\title{
INFERENCES OF PLASMA PARAMETERS FROM CORONAL HOLE OBSERVATIONS
}

\author{
SHADIA RIFAI HABBAL \\ Harvard-Smithsonian Center for Astrophysics
}

\begin{abstract}
The temperature in the acceleration region of the solar wind remains one of the most elusive parameters to measure. Knowledge of the temperature as well as its gradient in the inner corona is fundamental for placing constraints on physical mechanisms thought to be responsible for the coronal heating process, as well as for understanding the flow properties of the solar wind. Estimates of the helium abundance is essential for understanding the puzzling behavior of heavier ions in the solar wind. As an illustration of the difficulties and uncertainties involved in the inferences of plasma parameters in the solar wind acceleration region, the inference of electron temperature and helium abundance will be described. Prospects for future observations will be briefly discussed.
\end{abstract}

Key words: Temperature - Helium Abundance - Solar Wind

\section{Introduction}

Knowledge of the conditions in the acceleration region of the solar wind are essential for understanding the properties of the physical mechanisms responsible for its heating and acceleration. While in-situ observations have been very successful in yielding information about the solar wind beyond $0.3 \mathrm{AU}$, so far, the conditions within $0.3 \mathrm{AU}$ from the solar surface have been limited to remote observations. Since these measurements rely on the intensity of a variety of spectral lines as well as Thompson scattered white light, they carry information from a long integration path that intercepts plasmas with different properties. Consequently, there are inherent problems associated with these observations.

In this review, the primary emphasis is to point to some of the sources of uncertainties associated with inferences of plasma parameters in the acceleration region of the solar wind, namely in coronal holes. Electron temperature and helium abundance will be used as examples of such inferences.

\section{Observational characteristics of coronal holes}

So far, the remote imaging of the Sun has been confined to the ecliptic plane and to earth's orbit ( $1 \mathrm{AU})$. While the abundance of loop like structures is visible on the solar surface at all times, from this perspective, it is only close to solar minimum that coronal holes can readily manifest themselves. At EUV and X-ray wavelengths, they appear as regions of significantly reduced 
emission, whether observed directly against the solar disk, or against the plane of the sky (also referred to as "limb" observations). While disk observations show the predominance of small scale closed magnetic field structures referred to as coronal bright points [e.g. Habbal and Withbroe, 1979], limb observations show the existence of filamentary structures, originating from the bright points and extending outwards away from the solar surface. White light eclipse observations at solar minimum show how these filamentary structures, referred to as polar plumes, extend into interplanetary space, while remaining fairly well collimated [Koutchmy, 1977]. Interplanetary remnants of these filamentary structures have also been reported [Thieme et al., 1989; Woo, 1995].

\section{Inference of the electron temperature}

At present, our knowledge of the temperature gradient in coronal holes derives from a combination of a variety of observations, as summarized in Figure 1, none of which were made simultaneously, nor within the same region. The difficulty in establishing the temperature structure and temperature gradient within coronal holes from limb observations is due to the frequent veiling of the coronal holes by the hotter and denser quiet regions surrounding them and shaping their boundaries. An illustration of this effect is given in Figure 2, with two soft X-ray full disk images of the corona from Yohkoh [Tsuneta et al., 1991], taken 14 days apart. This example shows how the unobstructed extent of a coronal hole along the line of sight, which is extremely important for any measurement, is not always readily available, even when a coronal hole seems to be largely exposed on the disk (compare, for example the south polar coronal hole on those two days). While disk observations do not suffer from this problem, no height information can be obtained from them.

A summary of the different techniques commonly used for inferring electron temperatures is given in Habbal et al. [1993] (see also Mariska [1992]). Only the line ratio technique will be discussed here, since this approach is the most commonly used, and will be used with some of the instruments on SOHO [Domingo, 1989]. In this technique, the ratio of the measured intensity of a combination of spectral lines is used to infer the electron temperature, by comparing this ratio with the ratio of the emissivity of the corresponding lines computed theoretically. Given the temperature dependence of the emissivity, a temperature can be derived from the ratios. The basic assumption that enters this approach is that the plasma is homogeneous along the line of sight. Although not very realistic, this assumption is most reliable if the spectral lines used have a temperature response function with a large over- 


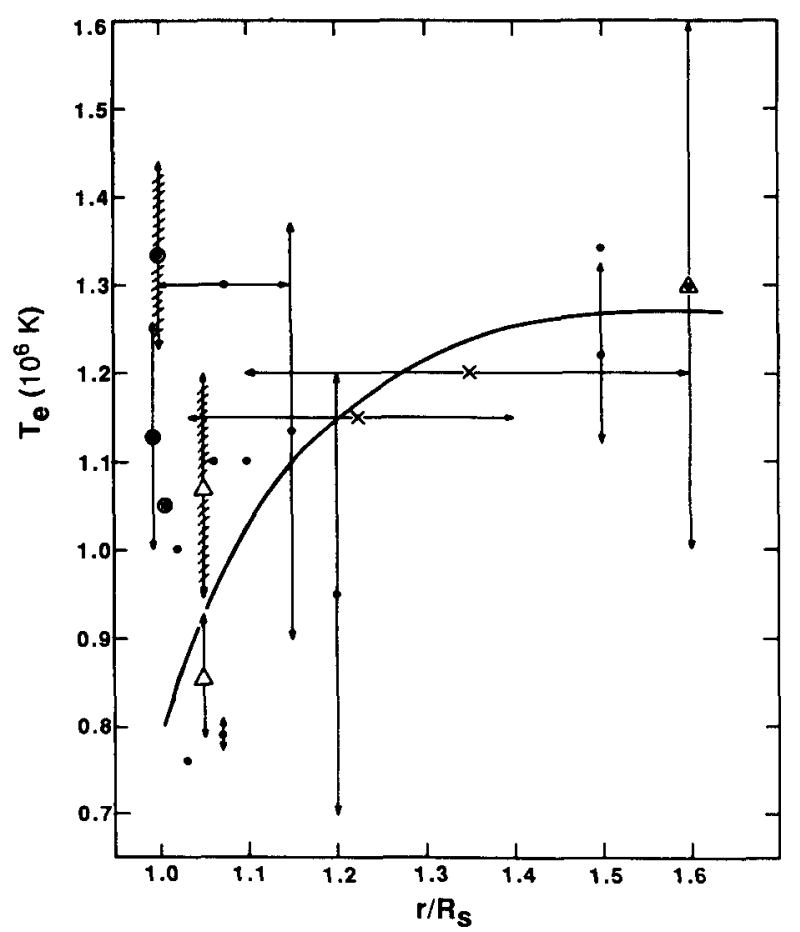

Fig. 1. Temperature gradient (solid curve) derived from different sets of empirical data: limb observations $(\bullet)$, white light $(x)$, disk observations $(\odot)$, in situ observations $(\triangle)$, $\Delta$ derived for a coronal hole. $\downarrow$ indicates the temperature range at a given heliocentric distance, while $\longleftrightarrow$ shows the radial extent of a given inferred temperature. The hatched arrows indicate measurements clearly made at and/or outside the boundaries of coronal holes. [From Habbal et al. 1993.]

lap. Another major uncertainty that affects this approach is the elernental abundance.

The inference of electron temperatures in coronal holes derived by $\mathrm{Habbal}$ et al. [1993] is given here as an example. These authors used line intensities of $\mathrm{Mg}$ X $625 \AA$, O VI $1032 \AA$, and Ne VII $465 \AA$ which were measured across a polar coronal hole by the EUV spectrometer on Skylab [Reeves et al., 1977]. To take into account the uncertainties in abundances, the empirical ratios were compared with the theoretically computed ratios for the range of abundances most commonly quoted in the literature, as shown in Figure 3. The electron temperature thus inferred is shown in Figure 4, together with the sequence of $\mathrm{Mg} \mathrm{X}$ images obtained with that instrument. As shown in the lower panel of Figure 4, there is a significant difference between the coronal hole temperature and its boundaries. Also worth noting is that some of the variations across the coronal hole could be due to structures within the 


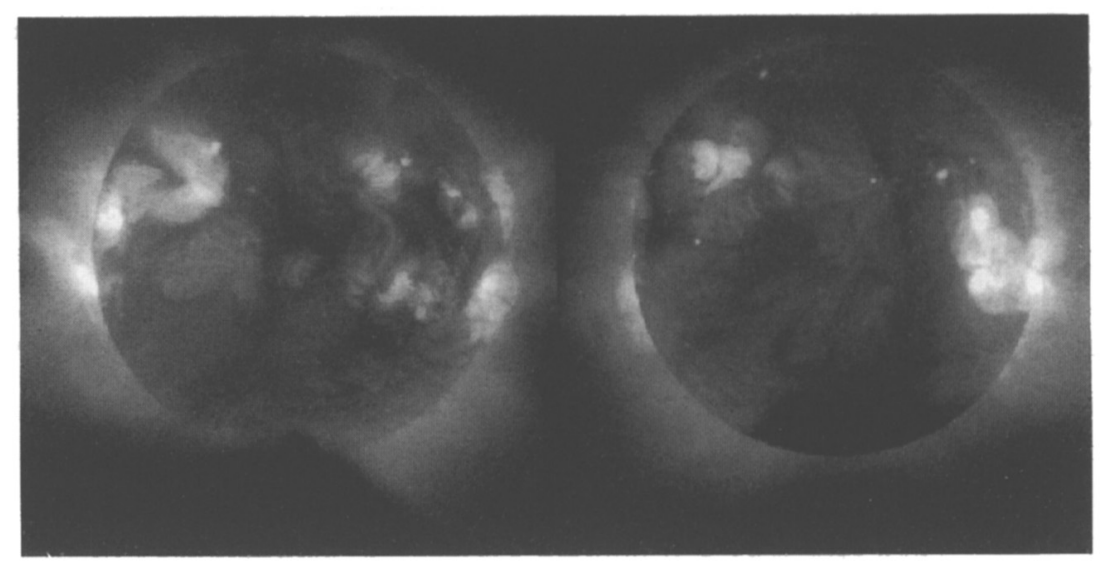

Fig. 2. Full disk images of the corona taken with the soft X-ray telescope on Yohkoh on 27 March 1993 (left) and 10 April 1993 (right) respectively.

region itself, such as polar plumes, or from foreground and/or background material. This example illustrates how the choice of spectral lines sensitive to cooler temperatures, i.e. at or below $10^{6} \mathrm{~K}$, is essential for probing coronal holes, and for minimizing the contribution from the hotter material that could be lying along the line of sight.

\section{Inference of the helium abundance}

The helium abundance, $\alpha$, defined as the ratio of the helium to proton densities, is observed to be remarkably constant around 0.05 at $1 \mathrm{AU}$ in high speed solar wind streams, while varying between 0.02 and 0.30 in slow speed winds [Bame et al., 1977]. On the other hand, theoretical considerations do not preclude the possibility that very large helium abundances, i.e. $>1$, could be present in the lower corona (see, e.g. Joselyn and Holzer [1978], Bürgi and Geiss [1986]).

So far, attempts to infer the helium abundance from observations in the inner corona have not been successful [e.g., Parkinson and Gabriel, 1986]. Recently, however, Habbal and Esser [1994] proposed a simple technique whereby limits on the helium abundance could be derived from knowledge of the electron temperature, density and their gradients. The approach is applicable very close to the coronal base, when the flow speed can be neglected 


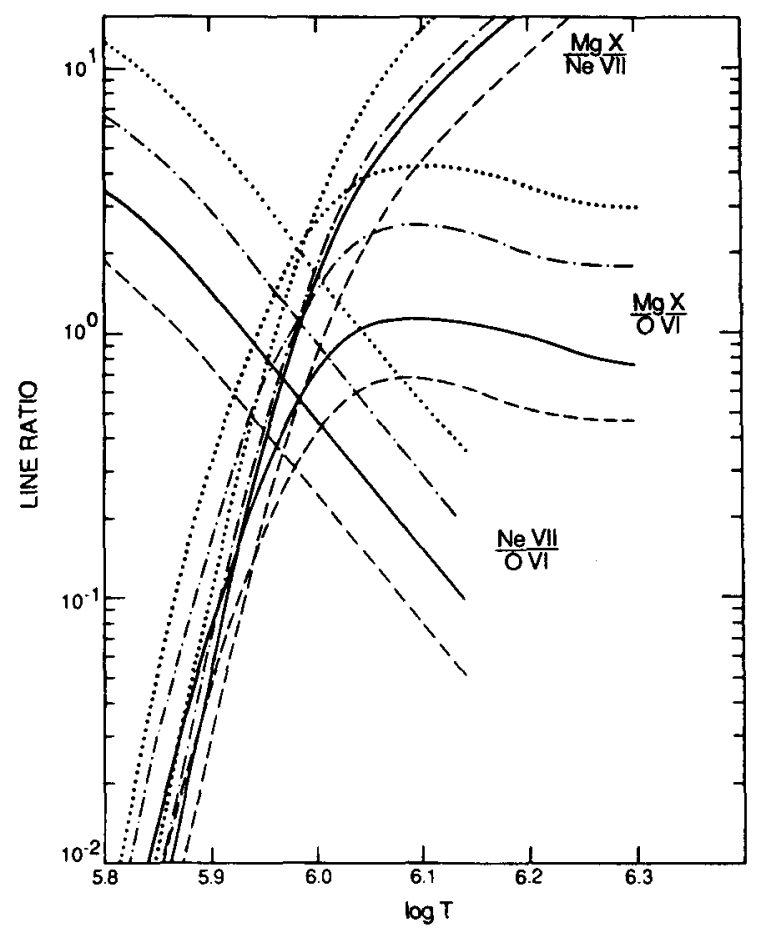

Fig. 3. Theoretical line ratios of $\mathrm{Mg} \mathrm{X} / \mathrm{Ne}$ VII, $\mathrm{Mg} \mathrm{X} / \mathrm{O} \mathrm{VI}$, and $\mathrm{Ne}$ VII/O VI computed for different combination of abundances. For ratios including $\mathrm{Mg} \mathrm{X}$, abundance combinations are: $\mathrm{Mg}=7.65, \mathrm{Ne}=7.92, \mathrm{O}=8.39$ (dotted lines); $\mathrm{Mg}=7.42, \mathrm{Ne}=7.92, \mathrm{O}=8.39$ (dash-dotted lines); $\mathrm{Mg}=7.65, \mathrm{Ne}=8.2, \mathrm{O}=8.96$ (solid lines) $\mathrm{Mg}=7.42, \mathrm{Ne}=8.2, \mathrm{O}$ $=8.96$ (dashed lines). For the $\mathrm{Ne}$ VII/O VI ratios the combinations are: $\mathrm{Ne}=8.2, \mathrm{O}=$ 8.39 (dotted); $\mathrm{Ne}=7.92, \mathrm{O}=8.39$ (dash-dotted); $\mathrm{Ne}=8.2, \mathrm{O}=8.96$ (solid); $\mathrm{Ne}=7.92$, $\mathrm{O}=8.96$ (dashed). [From Habbal et al., 1993].

in the force balance equation describing the three species. In this case, the momentum equation can be written in the form

$$
\frac{1}{n_{\alpha}} \frac{d n_{\alpha}}{d r}=\left(\frac{1+4 \alpha}{\alpha}\right) \frac{m_{p} G M_{s}}{k_{B} r^{2}} \frac{1}{T}+\left(\frac{2+3 \alpha}{\alpha}\right) \frac{1}{T} \frac{d T}{d r}+\frac{2(1+2 \alpha)}{\alpha} \frac{1}{n_{e}} \frac{d n_{e}}{d r}
$$

where $n_{\alpha}$ is the helium density, $n_{e}$ is the electron density, and $\mathrm{T}$ the temperature of the alpha particles, the electrons and the protons assumed to be the same.

Using empirical inferences of electron density and temperature and their gradients, together with the condition that $d n_{\alpha} / d r<0$, limits on the helium abundance can be derived as follows: For a given heliocentric distance, r, the variables and their gradients are substituted by their empirical values. One then searches for values of $\alpha$ for which the right hand side of Eq. (1) is negative. If $d T / d r<0$, a lower limit to the helium abundance is derived, while for $d T / d r>0$, one gets an upper limit. 

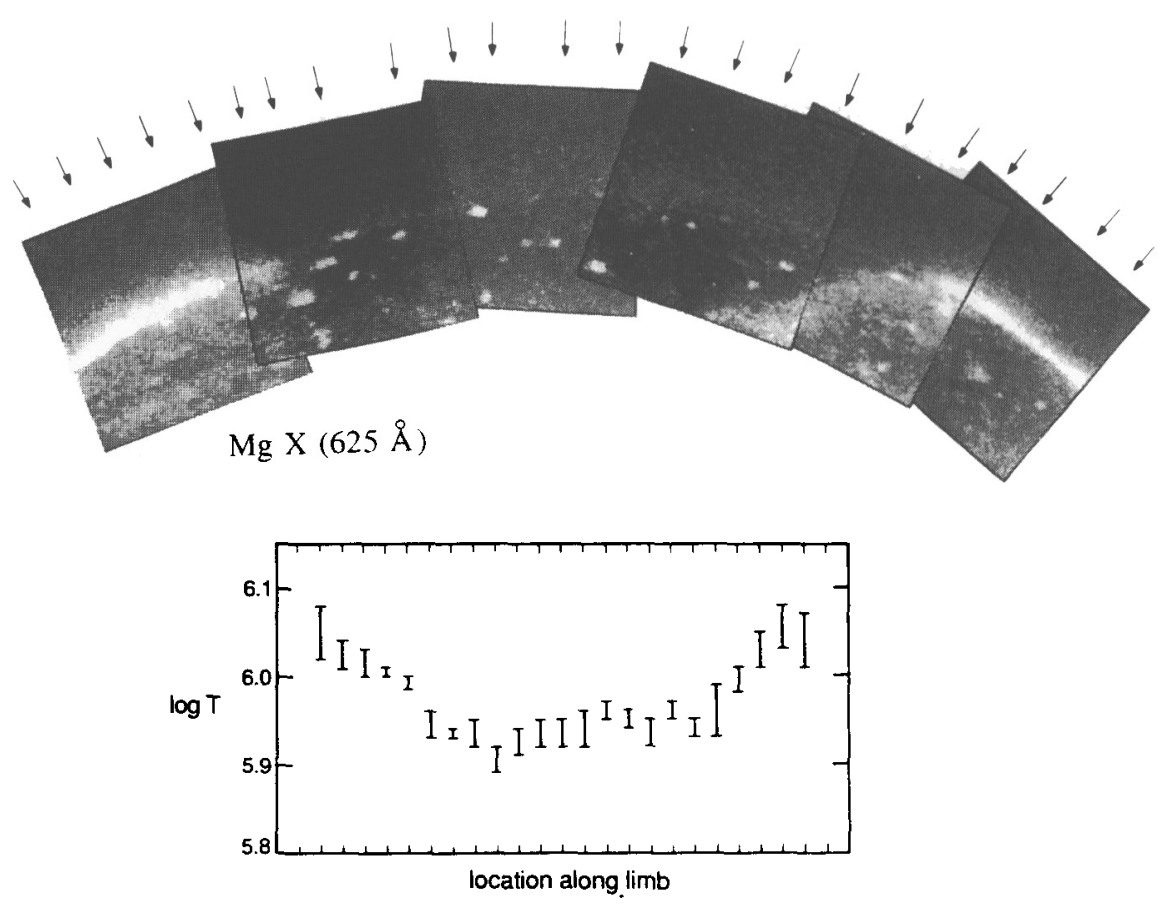

Fig. 4. Top panel: Composite of a south polar region as observed in $\mathrm{Mg} \mathrm{X}$ with the EUV/Skylab spectroheliometer on 1973 December 11, in a series of 5' $\times 5$ ' rasters. The arrows point to the direction where the intensities were computed. Lower panel: logarithm of the temperature as derived from the matching of the empirical line ratios of $\mathrm{Mg} \mathrm{X}$, $\mathrm{Ne}$ VII and O VI and the theoretical curves of Figure 3 for the following combination of abundances: $\mathrm{Mg}=7.65, \mathrm{Ne}=8.2$, and $\mathrm{O}=8.96$. [From Habbal et al., 1993].

Empirical values for the temperature gradient can be derived from the ratio of spectral line intensity measurements as described in the previous section. The density gradient can be inferred from white light observations. However, if only spectral line intensities are available, then the gradients of the emission measure can be used instead of the density gradient. In that case Eq. (1) can be replaced by

$$
\begin{aligned}
\frac{1}{n_{\alpha}} \frac{d n_{\alpha}}{d r}=-\frac{(1+\alpha)}{(1+3 \alpha)}\left[\frac{(1+4 \alpha)}{\alpha} \frac{m_{p} G M_{\mathrm{s}}}{k_{B} r^{2}}\right. & \frac{1}{T}+\frac{(2+3 \alpha)}{\alpha} \frac{1}{T} \frac{d T}{d r} \\
& \left.+\frac{(1+2 \alpha)}{\alpha(1+\alpha)}\left(\frac{1}{I_{\lambda}^{\prime}} \frac{d I_{\lambda}^{\prime}}{d r}-\frac{1}{L} \frac{d L}{d r}\right)\right]
\end{aligned}
$$

where $I_{\lambda}^{\prime}$ is the emission measure, and $L$ is the path length which contributes to the emission, and which, for the distances considered here (i.e. $<1.1 R_{s}$ ), can be assumed to be independent of $r$. 

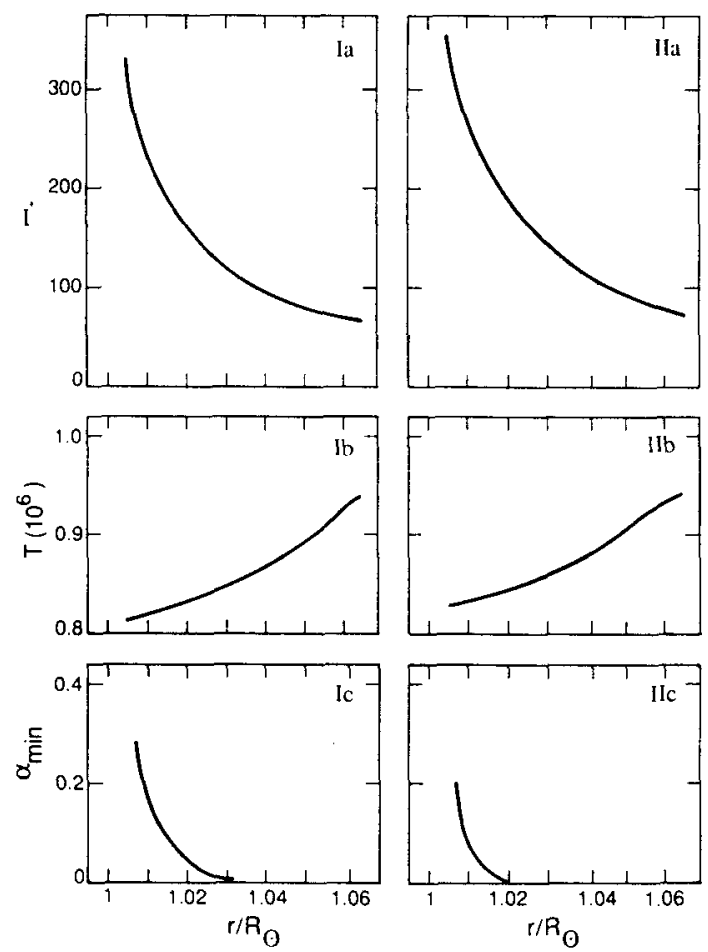

Fig. 5. Labels I and II refer to measurements and inferences along two different radial directions in a polar coronal hole. (a) Average emission measure, I', for the range of empirical values derived from emission line intensities, (b) average temperature profiles inferred from emission line ratios, (c) $\alpha_{\min }$ inferred from the data in (a) and (b) using the technique described in the text. No upper limit on $\alpha$ can be derived for these two examples. [From Habbal and Esser, 1994].

An example of the application of this technique to the polar coronal hole data shown in Figure 4 is given in Figure 5. This example indicates that the helium abundance, $\alpha$, can be at least 0.20 at the coronal base. This lower limit drops very rapidly to interplanetary values within a heliocentric distance of $1.1 \mathrm{R}_{\mathrm{s}} . \alpha_{\mathrm{min}}$ thus derived carries at least the same uncertainty entering the empirical derivation of the profiles for I' and $\mathrm{T}$, which are typically $25 \%$.

\section{Discussion and Conclusion}

Examples of the inference of the electron temperature and helium abundance in the inner corona, presented here, illustrate the difficulties associated with the inference of all plasma parameters from remote observations of coronal holes. Keeping those difficulties in mind, progress can be achieved with a 
judicious choice of spectral lines, and of observing times. The extent of coronal holes along the line of sight is a crucial factor to take into account, and can be best accomplished during solar minimum. As for the choice of spectral lines, the example presented here clearly indicates that electron temperatures in coronal holes are definitely cooler than their surroundings. Hence the choice of spectral lines for probing coronal holes should include lines formed primarily at or below $10^{6} \mathrm{~K}$. The advent of observations from different instruments on SOHO over the next few years offers one of the most promising opportunities at present. By combining adequate inferences with modeling one can hope to achieve a better understanding of the acceleration region of the solar wind.

The existence of structures within coronal holes is another aspect that should be taken into account in these inferences. So far, very little is known about the differences in plasma conditions between polar plumes and their surrounding. To explore their role in the solar wind flow, a clear distinction in their plasma properties has to be made.

Finally, the importance of coordinated multiwavelength observations has to be stressed. By combining simultaneous, yet different observing techniques, and different wavelength ranges, tight empirical constraints on crucial plasma parameters can be established.

\section{Acknowledgements}

This work was supported by NASA grants NAGW-249 and NAGW-3513. The author thanks Amy Mossman for her help with Figure 2.

\section{References}

Bame, S. T., et al.: 1977, J. Geophys. Res., 82, 1487.

Bürgi, A., and J. Geiss: 1986, Sol. Phys., 103, 347.

Domingo, V. (ed.): 1989, The SOHO Mission - Scientific and Technical Aspects of the Instruments, ESA SP-1104.

Habbal, S. R. and G. L. Withbroe: 1981, Solar Phys. 69, 77.

Habbal, S. R., R. Esser, and M. B. Arndt: 1993, Ap. J., 413, 435.

Habbal, S. R., and R. Esser: 1994, Ap. J., 421, L59.

Koutchmy, S.: 1977, Solar Phys., 51, 399.

Joselyn, J. A. and T. E. Holzer: 1978, J. Geophys. Res. 83, 1019.

Mariska, J. T.: 1992, The Solar Transition Region, Cambridge University Press.

Parkinson, J. H. and A. H. Gabriel: 1986, Adv. Space Res. 6 (8), 243.

Reeves, E. M., et al.: 1977, Appl. Optics, 16, 849.

Thieme, K. M., E. Marsch, and R. Schwenn: 1989, Adv. Space Res., 9, 127.

Tsuneta, S., et al.: 1991, Solar Phys., 136, 37.

Woo, R.: 1995, this volume. 